\title{
Mass composition studies using the surface detector of the Pierre Auger Observatory
}

\author{
Hernan Wahlberg ${ }^{a}$, for the Pierre Auger Collaboration

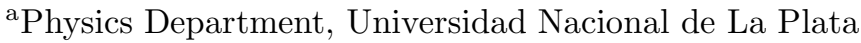 \\ C.C. 67-1900 La Plata, Argentina
}

The mass composition of ultra-high energy cosmic rays is a critical issue to understand their origin and nature. The Pierre Auger Observatory is a hybrid instrument which provides a powerful environment for the determination of the primary mass. The Surface Detector of the Pierre Auger Observatory alone allows the study of several shower parameters with high discriminating power between primary elements. Novel analysis techniques using different features of signals in the Cherenkov stations are discussed. These are the signal risetime, the azimuthal time asymmetry and the muon density of the showers.

\section{Introduction}

The determination of the nature of ultra high energy cosmic rays (UHECR) is at the moment an important issue towards the determination of their possible sources, the mechanism to acquire such high energies and their propagation. Differences between photon and neutrino induced showers present characteristics that easily separate them from hadronic shower [1,2]. However, the separation between hadron primaries (e.g. proton and iron ) is known to be complicated due to the large fluctuations resulting from the statistical nature of the shower development. Furthermore, the interpretation of data to determine mass composition has to be obtained by comparison with Monte Carlo predictions based on different hadronic models. With increasing cosmic ray energy, the gap between the shower energy and the energy range studied in accelerator experiments is filled by extrapolating known cross sections over a wide energy scale. Based on simple superposition models it can be shown that protons produce deeper showers with fluctuations larger than those of heavier nuclei. Therefore, for a given primary energy, the average depth of shower maximum $\left\langle X_{\max }\right\rangle$ and its fluctuations decrease with increasing primary mass. Heavier particles produce in addition more muons showing a higher muon to electromagnetic ratio.
The Surface Detector (SD) of the southern site of the Pierre Auger Observatory [3] consists of 1600 stations equally spaced on a triangular grid $(1.5 \mathrm{~km})$ over an area of approximately $3000 \mathrm{~km}^{2}$. Each SD station is a water Cherenkov tank, with digitalization acquisition electronics with a 40 $\mathrm{MHz}$ sampling rate. The Fluorescence Detector (FD) consists of 4 eyes with 6 telescopes each located at the border of the SD array overlooking it. The SD records the shower front, by sampling the particle density at ground level, with a duty cycle nearly $100 \%$. The FD measures the fluorescence light emitted as the shower develops through the atmosphere. As it can only operate on clear, moonless nights, its duty cycle is about $10 \%$. FD events provide a direct measurement of $X_{\max }$ [4] and at present is the main parameter to infer mass composition. However, the bulk of events collected by the Observatory have information only from the surface array and therefore observables from SD [5] as the one presented in this paper are important for composition analysis at the highest energies. Hadronic interaction models do not reproduce shower parameters in the same way, for example they show higher discrepancies in the number of muons than in the prediction of the shower maximum. Thus, comparing predictions with data for the longitudinal and lateral development of the showers should in 
addition, improve the constrains on the models.

\section{The risetime of the signal}

The first portion of the signal of the stations is dominated by the muon component which tends to arrive earlier and over a period of time shorter than the electromagnetic particles $(\mathrm{em})$, which are spread out on time.

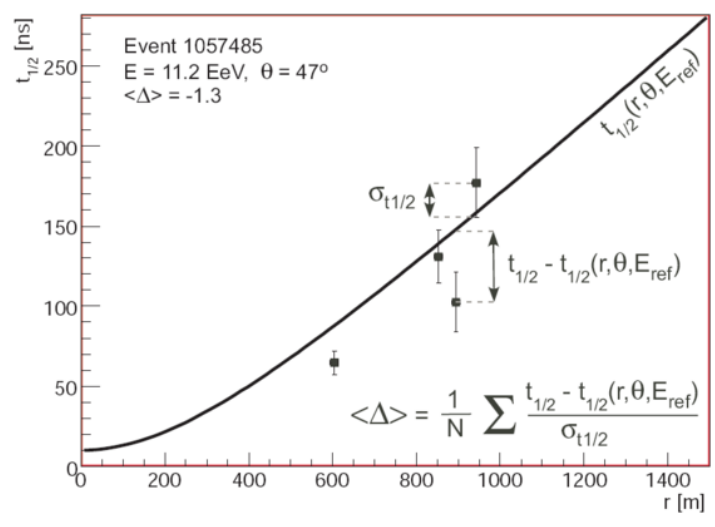

Figure 1. Ristime vs distance to the core. The curve is the benchmark risetime and the data points represent the measurements of ristime of each station with error bars for this particular event.

The risetime $\left(t_{1 / 2}\right)$ defined as the time to reach from $10 \%$ to $50 \%$ of the total integrated signal in each station, was shown to be effective for mass discrimination [6]. This is because it is sensitive to the $\mu$ to $\mathrm{em}$ ratio, a parameter that varies with the primary mass composition and is highly correlated with the shower development and the position of its maximum. A method was developed which consists on getting an averaged value of risetime (benchmark) found for the distance to the core $(r)$ and zenith angle $(\theta)$ for a given reference energy $\left(10^{19} \mathrm{eV}\right)$. Then, for each selected station, the deviation of the measured risetime from the benchmark is calculated in units of accuracy, and averaged for all stations in a given event as shown in equation 1 and Figure 1.

$$
<\Delta_{i}>=\frac{1}{N} \sum_{i=1}^{N} \frac{t_{1 / 2}-t_{1 / 2}^{\text {Benchmark }}(r, \theta)}{\sigma_{1 / 2}(\theta, r, S)} .
$$

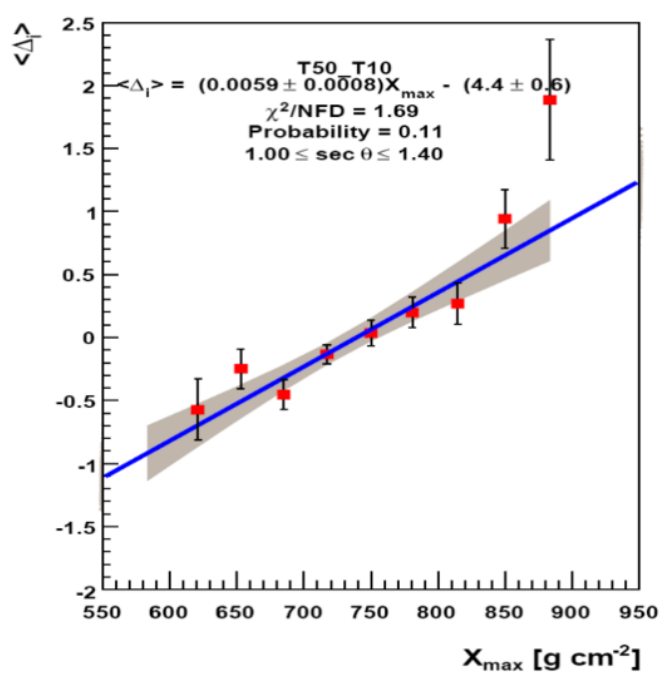

Figure 2. The average $\Delta_{i}$ as a function of $X_{\max }$ for the selected hybrid events. A correlation is seen which can be parameterized with a linear fit.

The $\left\langle\Delta_{i}\right\rangle$ should then be larger for showers developing deeper than the reference risetime (benchmark) and smaller for higher showers. Using hybrid events the parameter can be calibrated with $X_{\max }$. This is shown in Figure 2, where a linear dependence is found allowing an estimation of $X_{\max }$ from events observed by the SD alone. Mass composition studies can be done afterwards by means of the obtained elongation rate.

\section{Time asymmetry in the shower develop- ment}

The observed azimuthal asymmetry of time distributions from signals of inclined showers (incoming direction with zenith angle smaller than 
$\left.60^{\circ}\right)$ is a unique feature of the Pierre Auger Observatory which carries very valuable information related with the chemical composition of cosmic rays [7].

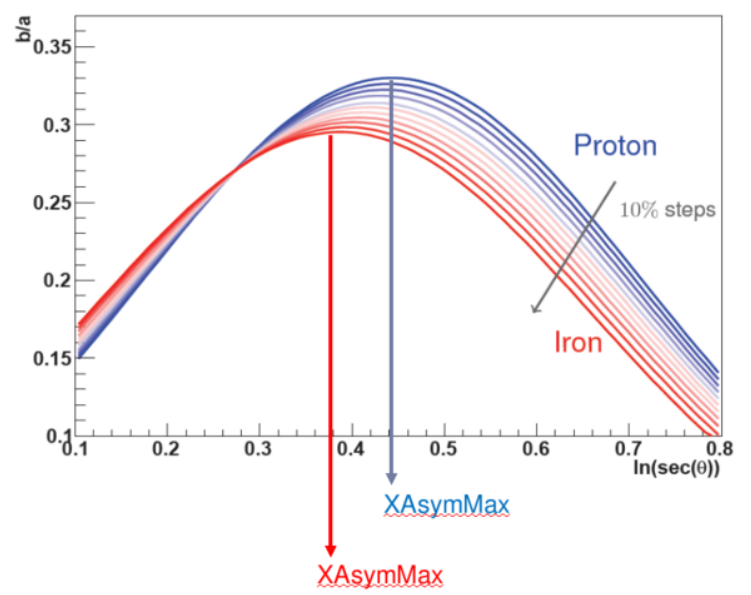

Figure 3. Asymmetry development for the different samples with mixed composition, going from pure proton to pure iron in steps of $10 \%$. The position of the maximum for the different primaries are explicitly marked.

The procedure used to get the average longitudinal development of the asymmetry, starts by selecting events in bins of reconstructed energy and $\sec \theta$. For these events the risetime of those stations passing the corresponding cuts is determined. Then for each $(E, \theta)$ bin, a fit of $<t_{1 / 2} / r>$ to a linear cosinus function of $\zeta$ (equation 2) provides the asymmetry factor $b / a$ :

$<t_{1 / 2} / r>\equiv \tau(r, \zeta)=a+b \cos \zeta$

where $\tau$ is the mean risetime divided by the distance to the core and $\zeta$ is the azimuth angle in the shower plane. The evolution of $b / a$ with zenith angle is an indicator of the shower development and is different for different primaries as shown in Figure 3.

In Figure 4 the values of the position of maximum asymmetry is represented against primary energy. Results for both hadronic models are included in the plots. The corresponding linear fits (continuous lines) of both primary types are clearly separated, thus allowing discrimination of heavy and light primaries.

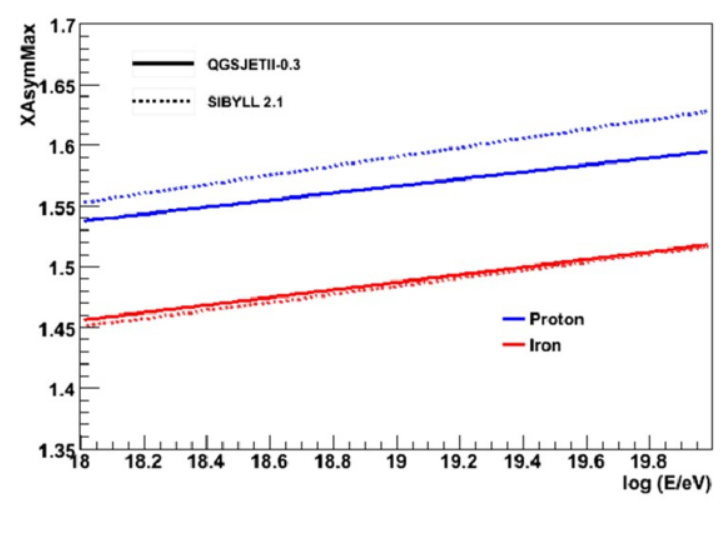

Figure 4. Position of maximum asymmetry vs. primary energy for different models and primaries. Lines correspond to fitted distributions of MC samples for proton and iron primaries.

\section{Muon Content}

The muon content of the EAS [8] is a quantity highly sensitive to mass composition. The FADCs of the SD detectors show a characteristic time structure from which the muon content can be measured. A typical individual muon deposits much more energy than the electromagnetic signal which looks more continuous and spread in time. Then muons should appear as isolated peaks above a continuous electromagnetic background. The FADC jump is defined at a time bin $t_{i}$ as:

$\Delta V\left(t_{i}\right)=V\left(t_{i+1}\right)-V\left(t_{i}\right)$

where $V\left(t_{i}\right)$ is the FADC signal. In Figure 5 the distribution of jumps $\frac{d N}{\Delta V}$ for real events is shown. The shape of the total signal can be described by the combined distribution of pure 
muonic and electromagnetic distributions. The number of muons is then estimated from the related area below $\frac{d N}{\Delta V}$ curve above a given threshold.

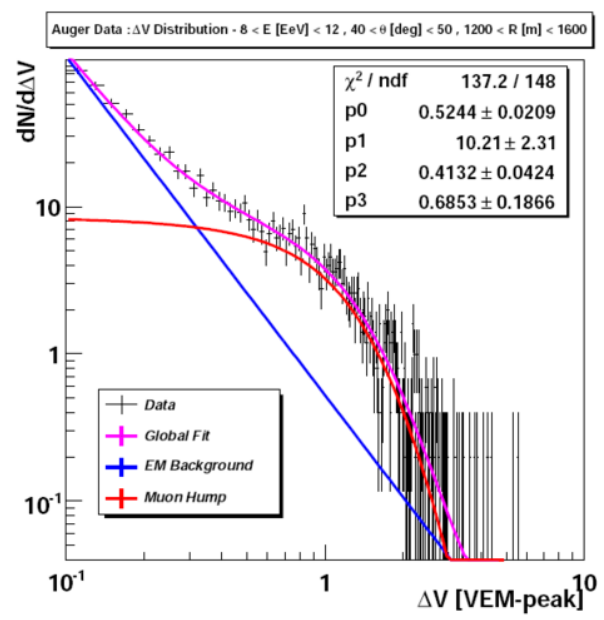

Figure 5. Distribution of jumps for events at a given energy, zenith angle and distance to the core range. Lines correspond to the different fitted distributions of muons and electromagnetic signal.

Figure 6 shows the muon density at 1000 meters from the core as a function of the primary energy of the incoming particle. The prediction of two different hadronic interaction models are shown for proton and iron primaries, showing differences between the two primaries and also the variations in the prediction of the different models. The absolute density and the slopes of the predictions can both out constrains to the models and infer composition trends.

\section{REFERENCES}

1. J. Abraham et al. [Pierre Auger Collaboration], Astropart. Phys. 29, 243 (2008)

2. J. Abraham et al. [Pierre Auger Collaboration], Phys. Rev. Lett. 100 (2008) 211101

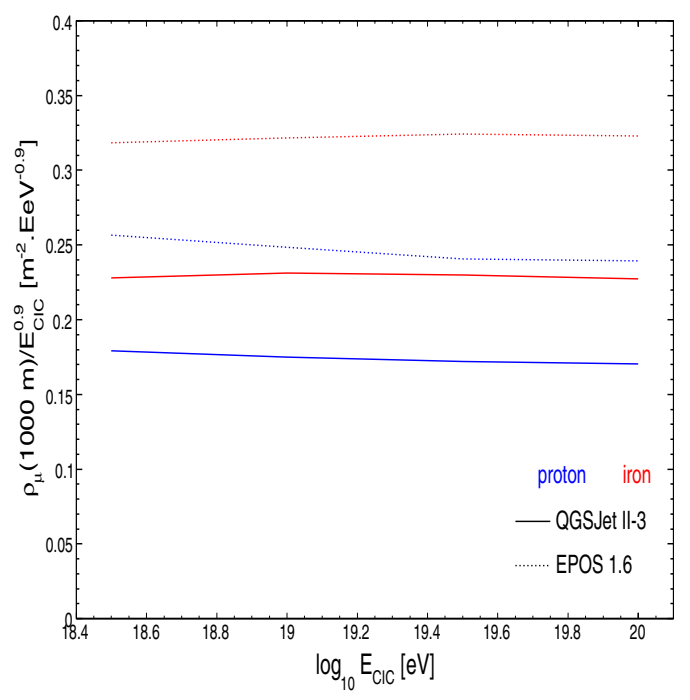

Figure 6. Muon density at 1000 meters from the core vs. energy of the primary particle for two different hadron interaction models. Lines correspond to the distributions of proton and iron simulations.

3. J. Abraham et al. [Pierre Auger Collaboration], Nucl. Instrum. Meth. A 523, 50 (2004).

4. M. Unger et al., Proc. 30th ICRC, Merida, Mexico (2007).

5. M. D. Healy et al.,Proc. 30th ICRC, Merida, Mexico (2007).

6. R. Walker \& A.A. Watson, J. Phys. G 7, 1279 (1981).

A.A. Watson. \& J.G.Wilson, J. Phys. A 7, 1199 (1974)

7. M. T. Dova et al., Proc. 29th ICRC, Pune, India, 00, 101-104 (2005).

8. F. Schmidt this proceeding. 